\title{
GUEST EDITORIAL Measuring up to high standards
}

\section{Pierre Karakiewicz}

Urological Oncologist, Assistant Professor, Director, Cancer Prognostics and Health Outcomes Unit, University of Montréal Health Centre, Montréal, Que.
$\mathrm{T}$ he current issue of CUAJ covers a variety of important topics. In the lead article, Nickel and colleagues provide the results of a survey of Canadian urologists about BPH visits as well as the diagnostic and treatment patterns. An average urologist only saw $15 \mathrm{BPH}$ patients weekly, despite the fact that most surveyed $(63 \%)$ practised in the community. The average initial visit IPSS was 14 and the overwhelming majority of men were treated medically: $44.7 \%, 17.9 \%$ and $11.1 \%$, respectively, received an $\alpha$-blocker, $5-\alpha$ reductase or both. A TURP was offered to an additional $5 \%$. The IPSS score and the rate of therapy are elevated. This may imply that urological referrals are predominantly made for symptomatic individuals, where medical therapy is required. The effect of therapy may be implied from the repeat visit IPSS 2-point decrease relative to the initial IPSS. Unfortunately, the relatively low proportion of participants ( 27 completed surveys v. 86 invited $=31.4 \%$ ) might be indicative of a participation bias, in which those more familiar and more interested in $\mathrm{BPH}$ may have been more likely to participate.

Mickelson and MacNeily provide expert insight about the CanMEDS project, which for the past decade has either elated or haunted those at academic institutions. Although some CanMEDS competencies (Medical Expert, Communicator and Scholar) pose no problems in our curricula, others (Collaborator, Manager, Health Advocate and Professionalism) may trigger a conceptual dilemma that might hinder their implementation and subsequent evaluation. These difficulties are compounded by busy academic practices, which leave little time and energy for the CanMEDS competencies, especially the more nebulous ones. Further difficulties stem from the lack of validation and (or) quantification of the benefits of the CanMEDS competencies on the overall health and (or) quality of care.

Finally, Dong and colleagues describe their institutional series of 77 consecutive laparoscopic pyeloplasties. The sample size and the quality of outcomes of this series are commendable, and outcomes exceed or at least parallel those from US centres of excellence. The significance of this Canadian report stems from many difficulties that Canadian urologists face in comparison with US urologists, especially for costly and technologically advanced surgical techniques (laparoscopy or robotics). Difficulties with developing Canadian surgical expertise in such fields originate from more limited patient volume relative to the United States as well as to healtheconomic barriers that dampen the rapid clinical implementation of modern medical technologies in Canada. Canadian laparoscopy and robotics have also clearly been affected by a more restricted patient pool and by the health-economic considerations, which make the achievement of equally successful outcomes substantially more difficult in Canada. Interinstitutional collaborations could circumvent some of the patient volume limitations and should be encouraged throughout the Canadian urological community. 